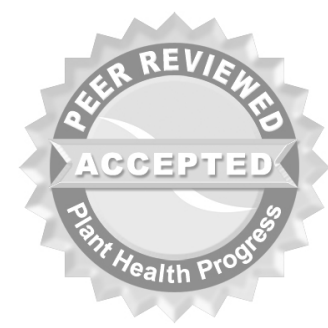

(c) 2011 Plant Management Network.

Accepted for publication 26 October 2011. Published 29 November 2011.

\title{
A Diagnostic Guide for Fusarium Wilt of Watermelon
}

Nathan M. Kleczewski, Postdoctoral Researcher, and Daniel S. Egel, Extension Plant Pathologist, Purdue University Department of Botany and Plant Pathology, Southwest Purdue Agricultural Center, 4369 N. Purdue Road, Vincennes IN 47591

Corresponding author: D. Egel. egel@purdue.edu

Kleczewski, N. M., and Egel, D. S. 2011. A diagnostic guide for Fusarium wilt of watermelon. Online. Plant Health Progress doi: 10.1094/PHP-2011-1129-01-DG.

Disease: Fusarium wilt

Hosts: Watermelon Citrullus lanatus (Thunb.) Matsum. \&Nakai

Pathogen: Fusarium oxysporum Schlechtend. :Fr. f.sp. niveum

Taxonomy

Kingdom Fungi; Division Ascomycota; Class Sordariomycetes; Order Hypocreales; Family Nectriaceae; Genus Fusarium; Species Fusarium oxysporum. Special form: niveum. Updated taxonomic placement can be found at www.cabi.org/ dfb and www.uniprot.org/taxonomy/ 5507 and information pertaining to taxonomy and evolution of the species is reviewed in Gordon and Martyn (11).

\section{Symptoms and Signs}

Fusarium wilt symptoms often start with a graying of foliage, followed by a general foliar chlorosis and wilt (Fig. 1A) (16). In older plants Fusarium wilt is characterized by wilting of individual watermelon runners (Fig. 1B). The hallmark of Fusarium wilt is unilateral stem necrosis, which is easily visualized when runners or stems are sectioned (Fig. 1C). Vascular necrosis is most prevalent at the soil line in older portions of the stem cut longitudinally. Under wet conditions white to pink fungal hyphae may be visualized emerging from necrotic tissues (Fig. 1D). Symptoms vary with FON race (21,35), density of fungal propagules (25), environment (30), and host genotype (8). Severely diseased plants may die within 10 days following infection (30) while plants colonized to a lesser extent may not express symptoms for several weeks. Growth of infected plants may be stunted without obvious wilt symptoms under some environmental conditions. 


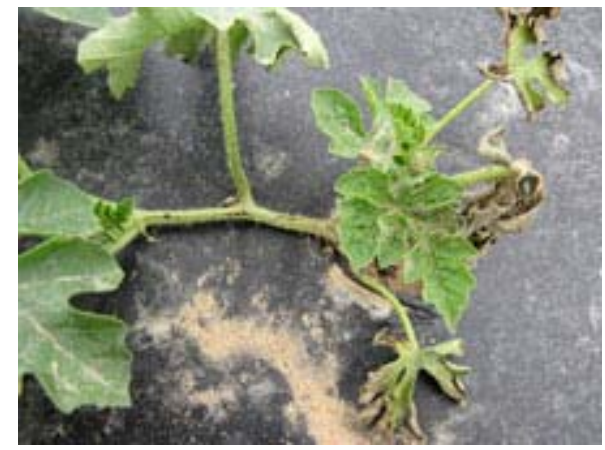

A
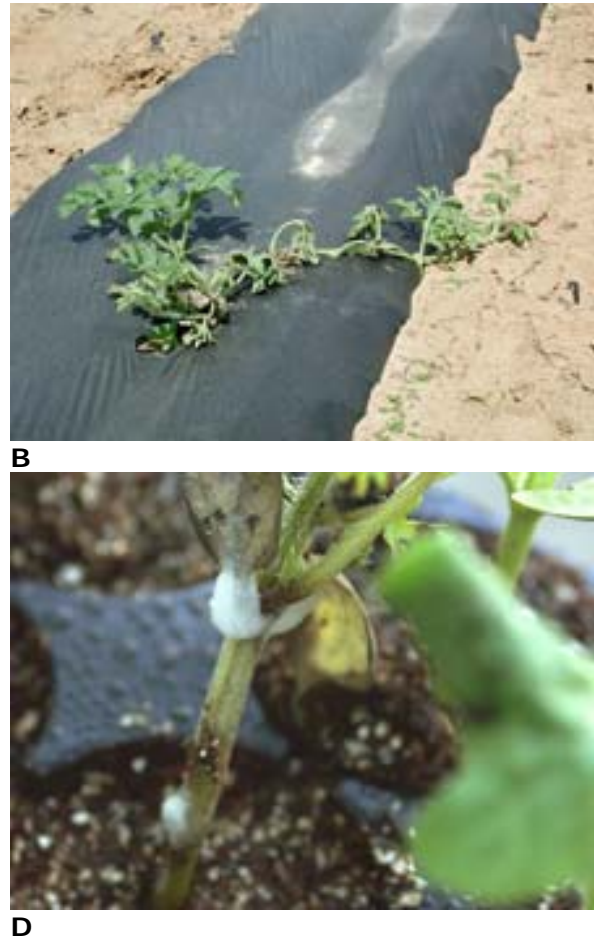

D

Fig. 1. Diagnostic symptoms of Fusarium wilt caused by Fusarium oxysporum f.sp niveum. (A) Foliage of infected plants is grey or chlorotic during early stages of the disease. (B) Fusarium wilt is most often observed in individual runners, not entire plants. (C) Infected stems are often necrotic when cross-sectioned. (D) Mycelia may be observed emerging from infected hypocotyl when humidy levels are high.

Soil factors influence symptom development of Fusarium wilt in watermelon. Soil temperature plays a strong role in determining disease severity $(13,31)$. Disease severity is greatest at soil temperatures between $25-27^{\circ}$ $\mathrm{C}$, and is greatly reduced above $27^{\circ} \mathrm{C}(23,31)$. In addition, disease severity is often increased with low soil $\mathrm{pH}$, reduced soil moisture, and high soil nitrogen levels $(12,14)$.

Greenhouse transplants may suffer from pre- or post-emergence damping off when infected with Fusarium oxysporum f.sp. niveum (FON) (15), either through infested growing media or contaminated seed $(2,24,27)$. Death of seedlings is rapid under appropriate conditions, and seedlings may die before the production of true leaves (Fig. 2A). The hypocotyl of infected seedlings is often noticeably necrotic (Fig. 2B). Foliage may also become chlorotic and necrotic prior to the seedling developing wilt. Asymptomatic transplants infected with low levels of FON may play a role in the spread of Fusarium wilt to uninfested fields (personal observation).

In the field, symptoms typically occur within 3 to 4 weeks, and may progress in asymptomatic plants after fruit set. Symptomatic vines may be clustered in the field based on pathogen population densities in the soil or soil type. Plants affected late in the season may wilt and collapse. Typically, not all vines in an area will be affected. Some symptoms of Fusarium wilt are shared with other diseases of watermelon. Therefore, it is essential that the pathogen isolation and identification protocols described in the subsequent sections are used to confirm the presence of FON. 

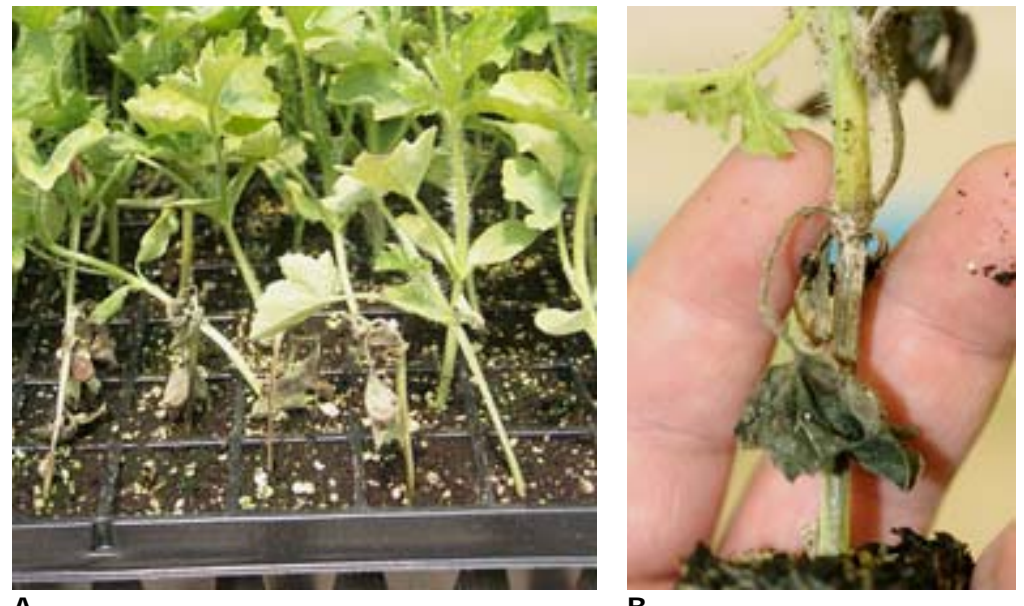

Fig. 2. Symptoms of Fusarium wilt in seedlings. (A) Post-emergancedamping-off can occur in watermelon seedlings exposed to Fusarium oxysporum f.sp. niveum (FON) in the greenhouse. (B) Basal stem necrosis is often observed in seedlings infected with FON (photograph by S. Saha, Purdue University Department of Horticulture).

\section{Host Range}

Fusarium oxysporum as a species contains over 100 special forms which infect only a particular host $(1,20)$. Consequently, FON is the special form that is pathogenic only on watermelon.

\section{Distribution}

Fusarium wilt is well established in nearly all watermelon growing regions of the world, and has been reported in Africa, Australia, Asia, Europe, North America, and South America. Detailed distribution maps for the pathogen can be viewed at: www.cabi.org/ dmpd. Distribution of the pathogen in the United States varies with race. Currently, four races of FON exist: races $0,1,2$, and 3 $(21,28,35)$. Race 3 is the most virulent race and causes wilt on watermelon lines resistant to other races of FON, but thus far has only been observed in Maryland (35). Race 2 is highly aggressive and has been isolated from wilted watermelon in Delaware (32), Indiana (7), Florida (22), Georgia (3), Maryland (32), Oklahoma (4), South Carolina (15), and Texas (21). Race 1 is the most widely distributed, and occurs throughout watermelon production areas of the United States (22). Traditionally, seeded, diploid-hybrid cultivars have good resistance to Race $1(8,33)$. The majority of watermelons have excellent resistance against race 0 , and the economic impacts of this race on watermelon are minor (22).

\section{Pathogen I solation}

If Fusarium wilt is suspected on watermelon, symptomatic plants or runners are cross-sectioned near the base of the crown. Isolation from fresh or newly diseased tissues minimizes contamination from secondary colonizers and saprophytic fungi or bacteria. Contamination may be further reduced by removing the vine cortex. Samples are rinsed under cool, running tap water to remove adhering soil and debris. Stem sections 1 to $2 \mathrm{~cm}$ in length are then surface disinfested in 0.6 to $1.2 \%$ active sodium hypochlorite (chlorine bleach) for one to five minutes, followed by 1 or 2 rinses in sterile water $(7,34)$. Sections are plated onto Komada's media, which is selective for F. oxysporum $(17,20)$. Other semi-selective media for Fusarium, such as PCNB-based media or Malachite-green media may also be used $(5,20)$; however, these media allow for other, non- Fusarium species to grow from tissues, which may complicate the identification of FON. Non-selective media such as PDA amended with streptomycin can also be used to isolate the pathogen, and may be useful if other wilt-inducing pathogens such as Rhizoctonia or Pythium are suspected (20). Plates are incubated at room temperature under 12 - $\mathrm{h}$ fluorescent lighting. Within five days, mycelium is visible growing out of the vascular bundles in infected sections (Fig. 3A) and will grow slowly on the selective media. 
Fusarium colony morphology varies widely, but isolates are often creamy orange to purple in culture (Fig. 3B). On PDA, Fusarium will grow rapidly, and mycelium may be floccose or sparse, but often fuzzier in appearance than on Komada's medium (Fig. 3B).

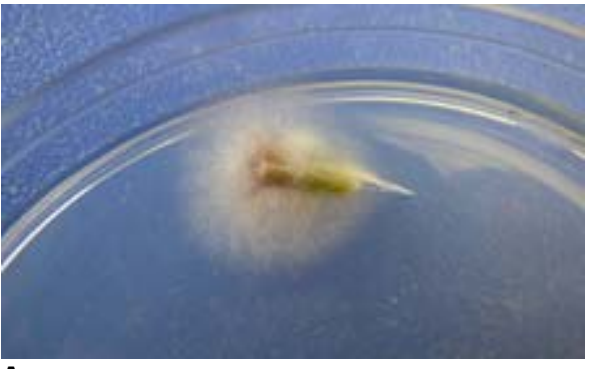

A

Fig. 3. I solation and growth of Fusarium oxysporum f.sp. niveum (FON) from symptomatic stems. (A) FON emerging from a surface-sterilized stem from a symptomatic watermelon seedling. (B) FON morphology varies with isolate and isolateion media. Here different FON race- 1 isolates have grown for 10 days on Komada's medium (upper row) and PDA (lower row).

To ensure isolate purity, dilution plating of microconidia onto Komada's medium should be done to produce multiple single spore individual colonies (20). Microconidia are produced by transferring 8 to $10,1-\mathrm{cm} \times 1$-cm blocks of pure cultures into 250-ml Erlenmeyer flasks containing 50 to $100 \mathrm{ml}$ of Esposito and Fletcher broth (9), followed by mild agitation on a rotary shaker for 3 to 5 days. Microconidial suspensions are diluted in sterilized water, and aliquots spread onto replicate petri plates containing Komada's media. Alternatively, a plug of pure culture FON may be transferred to a test tube containing sterilized tap water and vortexed to release conidia into solution. Aliquots of conidia are spread onto petri plates as described above. Individual microconidia that germinate are identified under a microscope and a single hyphal tip is excised to produce purified cultures.

\section{Pathogen I dentification}

For experienced researchers spore morphology is typically all that is required to identify F. oxysporum to species (20); however, there is no way to visually differentiate among the special forms or non-pathogenic F. oxysporum present in roots and crowns (18). Thus, screens should always be carried out to positively identify the pathogen. Single spore isolates should be transferred to carnation leaf agar (CLA) (20). On CLA and on infected tissues F. oxysporum will produce banana-shaped, often 3-septate macroconidia (Fig. 4A).

Macroconidia are produced on sporodochia, which are often located at the center of the petri plate. Sporodochia may not be present in all isolates. Small black/ purple sclerotia may also be present in some isolates. Small oval to kidney-shaped, often aseptate, microconidia (Fig. 4B) are produced on false heads on short monophialides formed on aerial mycelia. It may take over 4 weeks for some spore types and features to develop. Leslie and Summerell (20) provide an excellent diagnostic reference for Fusarium species (20). 


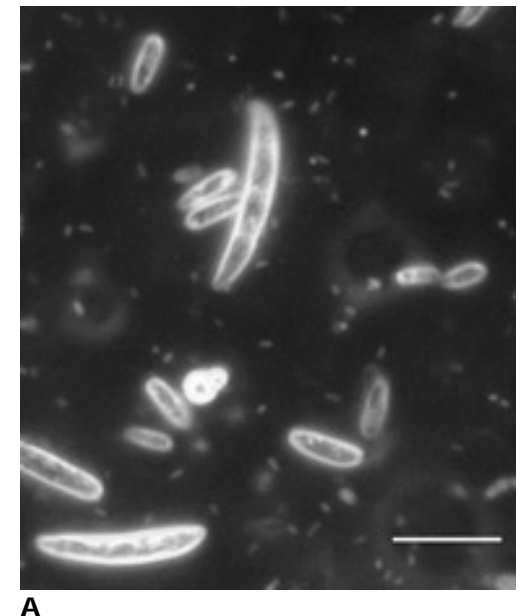

A

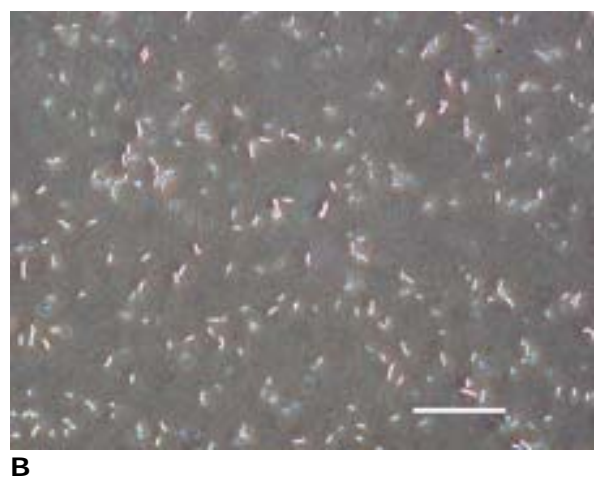

Fig. 4. Fusarium oxysporum produces macroconidia and microconidia.

(A) Macroconidia are boat shaped (fusiform), tapered, and septate. Scale

bar $=25 \mu \mathrm{m}$. (B) Microconidia are oval to kidney-shaped and usually aseptate.

Scale bar $=50 \mu \mathrm{m}$.

Molecular approaches can be used to complement morphological identification, and should be used when possible, particularly if the researcher has not previously worked with Fusarium. Many factors can influence spore morphology of Fusarium spp., potentially resulting in incorrect species designations based solely off of morphological characters (10). Researchers have developed a publicly available database (FUSARIUM-ID) of the partial translation elongation factor 1-alpha (TEF) DNA sequences for Fusarium spp., including F. oxysporum. The use of this region alone often results in correct placement of an isolate to species (10). The TEF region is amplified through PCR, using the ef1/ ef2 primer pair $(10,29)$. Once amplification of the TEF is confirmed, the $\sim 700 \mathrm{bp}$ DNA fragment is purified and sequenced in both directions to ensure high sequence quality. Sequences are then visually assessed using an appropriate DNA sequence editing program and compared to deposited sequences in the FUSARIUM-ID database using the basic local alignment search tool (BLAST) (10). Researchers that are trying to identify a new form species or race not previously identified in their area should use this technique to identify their Fusarium to species, and follow Koch's postulates to identify the special form. Morphological characterization then can be used to support the molecular data, increasing the reliability of the identification.

\section{Pathogen Storage}

A variety of methods can be used to store F. oxysporum. Freeze-drying or deep-freezing $\left(-80^{\circ} \mathrm{C}\right)$ cultures can preserve cultures for long periods of time (20). However, lyophilizers, liquid nitrogen, and $-80^{\circ} \mathrm{C}$ freezers may not be available to all researchers. A cheaper, more accessible method for long term storage of $\mathrm{F}$. oxysporum involves preservation on filter paper, followed by subsequent storage at $4^{\circ} \mathrm{C}(6)$. Autoclaved filter paper is laid on the surface of PDA in a 9-cm-diameter petri plate. An agar plug of F. oxysporum is transferred from a pure culture to the center of the filter paper, covered, and allowed to incubate under a lightbank with 12 -h lighting at room temperature. Once the fungus has grown within $1 \mathrm{~cm}$ of the filter paper edge, the filter is transferred aseptically to a sterile petri plate and allowed to dry for 2 to 3 days. Once dry, the filter paper is cut up axenically into small pieces $\sim 0.5 \mathrm{~cm} \times 0.5 \mathrm{~cm}$, transferred to sterile, $2-\mathrm{ml}$ screwtop vials, and stored at $4^{\circ} \mathrm{C}$. Individual filter paper squares may be used to start new cultures for future experiments (6). We routinely utilize this method and have successfully revived FON cultures over 10 years old.

\section{Pathogenicity Tests}

Pathogenicity tests must be conducted to positively determine that the suspect isolate of F. oxysporum is pathogenic to watermelon. Basic 
pathogenicity screens should be carried out on highly susceptible cultivars (e.g., Sugar Baby or Black Diamond) (21). Seedlings are typically grown from seed in pasteurized or autoclaved sand, potting media, vermiculite, or perlite $(19,21,34)$. Potting media containing microbes such as Trichoderma or Vesicular Arbuscular Mycorrhizal fungi should not be used as these microbes may reduce or prevent colonization by F. oxysporum. Once seedlings have developed 1 or 2 true leaves, they are removed from the media and carefully rinsed under cool running water. Seedlings that are older than the 2-leaf stage are less susceptible to FON and should not be used because susceptibility to FON decreases as seedlings age. A suspension of $\mathrm{F}$. oxysporum microconidia is prepared using Esposito and Fletcher broth as described previously, and adjusted to a concentration of $1 \times 10^{5}$ or $1 \times 10^{6}$ spores / $\mathrm{ml}$. The root systems for at least ten replicate seedlings are dipped in the spore suspension or the spore suspension is pipetted directly onto bare roots (19). Care should be taken to ensure that the length of time that the roots are dipped into the spore solution or the volume pippetted to roots is similar between tests and plants to reduce confounding factors. The seedlings are carefully transplanted into pots containing 4:1:1 (vol:vol:vol) sand:vermiculite:peat. Replicate seedlings with roots dipped in Esposito and Fletcher broth, which does not contain the isolate, should be used as negative controls. Plants should be transferred to a controlled environment at soil temperatures between 24 and $27^{\circ} \mathrm{C}$. Soil temperatures above $30^{\circ} \mathrm{C}$ significantly reduce FON infection and may result in the researcher incorrectly concluding that an isolate is not FON. Symptoms of wilt will often appear within 10 to 15 days, but timing varies with environment, aggressiveness of the isolate and size of seedlings at inoculation. Alternatively, seeds can be sown directly into pots containing the sand:vermiculite:peat mix and spore suspensions (at least $50 \mathrm{ml}$ ) added a rate of at least 2000 to 3000 spores/g soil to ensure that the microconidia will come into contact with the root system. This method is simpler, but results may be more variable in comparison to the root-dipping technique. Re-isolation of the fungus from sections of diseased plants and plating of sections of control plants should be conducted as previously described onto Komada's medium to complete Koch's postulates. All pathogenicity tests should be carried out at least twice to confirm results.

Pathogen race can be determined on a set of watermelon lines that vary in their level of resistance to F. oxysporum $\mathrm{f}$. sp niveum, although race determination can be challenging even for the most experienced of researchers. The differential set of plants should include Sugar Baby, Charlston Grey, Calhoun Grey, and PI-296341-FR (26). Based on this set, race 0 will only cause wilt on Sugar Baby, race 1 will wilt Sugar Baby and Charlston Grey, race 2 will wilt on Sugar Baby, Charlston Grey, and Calhoun Grey, and Race 3 will wilt all four differentials (35). Calhoun Grey is no longer readily available and may be substituted by Allsweet or Dixielee (33). The methods described in the previous section are used to prepare seedlings, media, and FON microconidia. All new isolates must be tested along with known isolates of races $0,1,2$, and 3 as positive controls. As mentioned in the previous section, seedlings must be no older than the 2-leaf stage, care must be taken to ensure that soil temperatures are optimal for FON infection, and spore applications must be uniform between plants and experimental replications. A failure to follow these guidelines may result in incorrect race assessment. To ensure accurate results, a minimum of 10 seedlings should be tested per repetition and a minimum of three repetitions conducted for each isolate. Wilt incidence levels less than 33\% should be considered resistant (35).

\section{Literature Cited}

1. Armstrong, G. M., and Armstrong, J . K. 1978. Formae speciales and races of Fusarium oxysporum causing wilts of cucurbitaceae. Phytopathology 68:19-28.

2. Boughalleb, N. and El Mahjoub, M. 2006. In vitro determination of Fusarium spp. infection on watermelon seeds and their localization. Plant Pathol. J . 5:178-182.

3. Bruton, B. D., Fish, W. W., and Langston, D. B. 2008. First report of Fusarium wilt caused by Fusarium oxysporum $\mathrm{f}$. sp niveum race 2 in Georgia watermelons. Plant Dis. 92:983-983. 
4. Bruton, B. D., Patterson, C. L., and Martyn, R. D. 1988. Fusarium wilt (F. oxysporum f.sp. niveum race-2) of watermelon in Oklahoma. Plant Dis. 72:734734.

5. Castellá, G., Bragulat, M. R., Rubiales, M. V., and Cabañes, F. J . 1997. Malachite green agar, a new selective medium for Fusarium spp. Mycopathologia 137:173178.

6. Egel, D., and Hoke, S. 2009. Long term refrigerator storage of Didymella bryoniae and other fungi on filter paper. Nat. Plant Diag. Net. 4:5-6.

7. Egel, D. S., Harikrishnan, R., and Martyn, R. D. 2005. First report of Fusarium oxysporum f. sp. niveum race 2 as causal agent of Fusarium wilt of watermelon in Indiana. Plant Dis. 89:108-108.

8. Elmstrom, G. W., and Hopkins, D. L. 1981. Resistance of watermelon cultivars to Fusarium wilt. Plant Dis. 65:825-827.

9. Esposito, R. G., and Fletcher, A. M. 1961. Relationship of pteridine biosynthesis to action of copper 8-hydroxyquinolate on fungal spores. Arch. Biochem. Biophys. 93:369-376.

10. Geiser. D.M. del MarJ imenez-Gasco, M., Kang, S., Makalowska, I., Veeraraghavan, N., Ward, T. J., Zhang, N., Kuldau, G. A., and O'Donnell, K. 2004. FUSARIUM-ID v. 1.0: A DNA sequence database for identifying Fusarium. Euro. J . Plant Pathol. 110:473-479.

11. Gordon, T. R., and Martyn, R. D. 1997. The evolutionary biology of Fusarium oxysporum. Ann. Rev. Phytopathol. 35:111-128.

12. Hopkins, D. L., and Elmstrom, G. W. 1976. Effect of soil pH and nitrogen source on Fusarium wilt of watermelon on land previously cropped in watermelons. P. Florida State Hortic. Soc. 89:141-143.

13. Huang, J., and Sun, S. 1978. Factors affecting survival of watermelon wilt pathogen, Fusarium oxysporum (S.Chl.) f.sp. niveum (E.F. Sm) Snyder + Hansen, in soils. Plant Prot. Bull. 20:56-66.

14. J ones, J. P., Woltz, S. S., and Everett, P. H. 1975. Effect of liming and nitrogen source on Fusarium wilt of cucumber and watermelon. P. Florida State Hortic. Soc. 88:200-203.

15. Keinath, A. P., and DuBose, V. 2009. First report of Fusarium oxysporum f. sp. niveum race 2 in South Carolina watermelon fields. Phytopathology 99:S63-S63.

16. Koike, S. T., Gladders, P., and Paulus, A. O. 2007. Vegetable Diseases: A Color Handbook. Academic Press, Burlington, MA.

17. Komada, H. 1976. A new selective medium for isolating fusarium from natural soil. P. Am. Phytopathol. Soc. 3:(1977).

18. Larkin, R. P., and Fravel, D. R. 1999. Mechanisms of action and dose-response relationships governing biological control of Fusarium wilt of tomato by nonpathogenic Fusarium spp. Phytopathology 89:1152-1161.

19. Latin, L. X., and Snell, S. J. 1986. Comparison of methods for inoculation of muskmelon with Fusarium oxysporum f.sp. melonis. Plant Dis. 70:297-300.

20. Leslie, J. F., and Summerell, B. A. 2006. The Fusarium Laboratory Manual. Blackwell Publishing, Victoria, Australia.

21. Martyn, R. D. 1987. Fusarium oxysporum f.sp. niveum race-2: A highly aggressive race new to the United States. Plant Dis. 71:233-236.

22. Martyn, R. D., and Bruton, B. D. 1989. An initial survey of the United States for races of Fusarium oxysporum f.sp. niveum. Hortscience 24:696-698.

23. Martyn, R. D., and Hartz, T. K. 1986. Use of soil solarization to control Fusarium wilt of watermelon. Plant Dis. 70:762-766.

24. Martyn, R. D., and McLaughlin, R. J . 1982. Identification and pathogenicity of Fusarium species isolated from surface-disinfested watermelon seed. J. Seed Technol. 7:97-107.

25. Martyn, R. D., and McLaughlin, R. J. 1983. Effects of inoculum concentration on the apparent resistance of watermelons to Fusarium oxysporum f.sp. niveum. Plant Dis. 67:493-495.

26. Martyn, R. D., and Netzer, D. 1991. Resistance to races 0,1, and 2 of Fusarium wilt of watermelon in Citrullus sp. PI-296341-FR. Hortscience 26:429-432.

27. Michail, S. H., Rehim, M. A., Tarabeih, A. M., and Aly, M. A. 2002. Effect of Fusarium seed-borne infection levels on watermelon wilt incidence. Acta Phytopathologica et Entomologica Hungarica 37:347-351.

28. Netzer, D. 1976. Physiological races and soil population level of Fusarium oxysporum f.sp. niveum wilt of watermelon. Phytoparasitica 4:131-136.

29. O’Donnell, K., Kistler, H. C., Ciglnik, E., and Ploetz, R. C. 1998. Multiple evolutionary origins of the fungus causing Panama disease of banana: Concordant evidence from nuclear and mitocondrial gene genealogies. Proceedings of the National Academy of Sciences of the United States of America 95:2044-2049. 
30. Porter, D. R. 1932. Some environmental relations of watermelon wilt. Phytopathology 22:813-825.

31. Walker, M.N. 1941. Fusarium wilt of watermelons. I. Effect of soil temperature on the wilt disease and the growth of watermelon seedlings. Florida Agricultural Experiment Station 363:1-29.

32. Zhou, X. G., and Everts, K. L. 2001. First report of the occurence of Fusarium oxysporum f.sp. niveum race 2 in commercial watermelon production areas of Maryland and Delaware. Plant Dis. 87:692-698.

33. Zhou, X. G. and Everts, K. L. 2003. Races and inoculum density of Fusarium oxysporum f. sp. niveum in commercial watermelon fields in Maryland and Delaware. Plant Dis. 87:692-698.

34. Zhou, X. G., and Everts, K. L. 2004. Quantification of root and stem colonization of watermelon by Fusarium oxysporum $\mathrm{f}$. sp niveum and its use in evaluating resistance. Phytopathology 94:832-841.

35. Zhou, X. G., Everts, K. L., and Bruton, B. D. 2010. Race 3, a new and highly virulent race of Fusarium oxysporum $\mathrm{f}$. sp niveum causing Fusarium wilt in watermelon. Plant Dis. 94:92-98. 